\title{
Measures of The Direct Impact of Hematophagous Flies On Feeder Cattle: An Unexpectedly High Potential Economic Impact
}

Phoompong - Boonsaen

Kasetsart University Kamphaeng Saen Campus

\section{Adèle Nevot}

UMR INTERTRYP: Unite Mixte de Recherche Interactions Hote-Vecteur-Parasite-Environnement dans les maladies tropicales negligees dues aux Trypanosomatidae

Sathaporn Onju

Kasetsart University Kamphaeng Saen Campus

Clément Fossaert

CIRAD

\section{Piangjai Chalermwong}

Kasetsart University - Bangkhen Campus: Kasetsart University

Kornkanok Thaisungnoen

Kasetsart University - Bangkhen Campus: Kasetsart University

Antoine Lucas

CIRAD

\section{Sophie Thévenon}

CIRAD

Roungthip Masmeatathip

Kasetsart University Kamphaeng Saen Campus

Sathaporn Jittapalapong ( $\nabla$ sjittapalapong@gmail.com )

Kasetsart University https://orcid.org/0000-0001-6566-8050

\section{Marc Desquesnes}

CIRAD

\section{Research Article}

Keywords: biting flies, mosquitoes, annoyance, production loss, weight gain

Posted Date: July 27th, 2021

DOI: https://doi.org/10.21203/rs.3.rs-717630/v1 
License: (c) (i) This work is licensed under a Creative Commons Attribution 4.0 International License. Read Full License 


\section{Abstract}

Beside blood pathogens transmission, insects of the order Diptera affect livestock by visual and contact harassment; blood-feeders are responsible of painful bites and blood despoliation, generating behavioural modifications, anaemia and production losses. Knowledge of their economic impact is a basis for cost-effective control. Here, we measured the global impact of Diptera by comparing two batches of six feeder cattle, one in open air, the other protected by a mosquito net. Analytical data were: insect density in open air, and, for feeder cattle: tail-flick counts, hematocrit values $(\mathrm{Ht})$, feed intake, feed conversion ratio (FCR) and live body weight gains (LBWG). Over a period of five months, results showed significant losses of LBWG of cattle exposed to insects, estimated at $8.0 \pm 1.5 \mathrm{Kg} / \mathrm{month}[2.7 ; 13.3]$, with a total loss reaching $40.0 \pm 5.5 \mathrm{Kg} / \mathrm{head}$. Main diurnal insects were Stomoxys spp. and M. crassirostris. There was a strong correlation between fly density and diurnal tail-flicks. Night trapping and tail-flicks showed a potentially important role of mosquitoes to be further explored. Ht of exposed animals was 3$4 \%$ lower than controls. FCRs indicated that exposed animals needed $33 \%$ more dry matter intake $/ \mathrm{Kg}$ of LBWG. Economic assessment showed that dipterans were responsible of 10-11\% loss of LBWG during the main growing period of feeder-cattle (10-15 months). A feedlot of 100 calves would register a total loss of 16,000 within 5 months, which appears to be an unexpectedly huge loss caused by dipterans. Investing part of this money in fly control would probably be beneficial.

\section{Introduction}

Insects belonging to the order Diptera are the most important pest of livestock and humans; amongst them, some are pest at the larval stage, the agents of myiases, such as Oestridae, Sarcophagidae, Calliphoridae etc., but the most important are pest at the adult stage (Duvallet 2017). Common flies such as Musca and Fannia spp. are annoying by their simple presence when they are in large numbers, but the negative impact of flies is mostly due to blood sucking insects, which are especially proliferating under massive monoculture production (El-Laithy 2013).

A large range of hematophagous insects are considered as pests of livestock; the most important belong to the Order Diptera, sub-orders Brachycera and Nematocera. In the suborder Brachycera are the highly abundant hematophagous biting flies, such as stomoxyine flies, tabanids, tsetse flies, hippoboscids, but also the common sponging flies. Beside this, in the suborder Nematocera, are the very common and abundant mosquitoes (Culicidae), together with culicoides, phlebotomes and simulies (Duvallet 2017).

Amongst these insects, the most prevalent livestock dipteran pests are sponging flies of the genus Musca (some of them being obligatory hematophagous such as Musca ventrosa, M. inferior and M. crassirostris (Patton 1922, Desquesnes et al. 2018)), stomoxyine flies, especially those belonging to the genus Stomoxys (stable flies), and tabanids and mosquitoes. Not only acting as direct pests, these insects are also responsible of biological or mechanical transmission of a large range of pathogens, including bacteria, viruses and parasites (Baldacchino et al. 2013, Baldacchino et al. 2014). 
Protection of livestock under a mosquito net is an efficient option to avoid the annoyance of these insects, however, it is generally difficult to maintain in a farm, due to human and animal movements needed for feed provision, pen cleaning and other managements. Permanent protection under a mosquito net may be applied under zero grazing conditions (Bauer et al. 2006). Even though, it is rarely sustainable for the reasons mentioned above. Killing the insects coming into contact with livestock by the use of insecticides applied by pour-on preparation, spray or injection, is another option; it is costly and polluting, by spreading insecticides both in animal products and environment. Treatment frequency required may reach 2-3 times a week for insecticide sprays in the control of tabanid or Stomoxys infestations (Bruce and Decker 1951a, Raymond and Favre 1991). These measures are generally of low efficacy, and they are not sustainable (Saliba et al. 1976). Other means such as traps and/or impregnated screens have been used but not widely so far (Hogsette et al. 2008); new polyethylene screens have recently been developed to attract and kill tabanids and stomoxes (Desquesnes et al. 2019), however, demonstrating their costefficiency will be necessary.

Unless bred indoor and/or under permanent mosquito net protection, livestock are permanently submitted to the annoyance, the bite, and the blood despoliation inflicted by diurnal and nocturnal hematophagous insects that impaired seriously livestock production. The total impact of these insects on livestock has never been determined, however, based on partial data available and estimations made for some types of insects such as Stomoxys spp. and tabanids, it is generally accepted that biting flies are responsible of a huge loss in livestock production. In a very early study made in 1947 in Illinois, US, it was estimated that controlling tabanids through insecticide spray (every 3-4 days) might bring an additional weight gain of 9-13.5 Kg per 5.5 weeks in free pasturing cattle (Bruce and Decker 1951a, Bruce and Decker 1951b). In a study made in USSR in 1982-84, it was estimated that tabanids were responsible of a decrease of $13 \%$ of dairy production; at that time only insecticide spray was proposed to decrease their impact (Minár et al. 1987). In French Guyana, in 1992, the daily weight gain of feeder cattle registered during the tabanids' infestation season decreased from an average of $1,200 \mathrm{~g}$ down to $200 \mathrm{~g}$; at the same period, the economic activity of horse-riding was totally interrupted for 2-3 months, during tabanids' season (Desquesnes 2004). Stomoxyine flies such as Hematobia (horn flies) and Stomoxys (stable flies) are sometimes considered as responsible for a loss of $50 \%$ of milk production (Hill 1997), and for important loss of weight-gain in feeder cattle (Wiemann et al. 1992). Other studies in the US emphasized on the predominant impact of horn flies in cattle, including beef and dairy cattle; an increase of $12.5 \mathrm{Kg}$ was obtained in calf weight on weaning in insecticide-protected animals versus control group (Byford et al. 1992). In another study carried out in the US, the losses due to stable flies were estimated per animal and year at $139 \mathrm{~kg}$ of milk for dairy cows, and 6, 26, and $9 \mathrm{~kg}$ of live body weight for pre-weaning calves, pastured stockers, and feeder cattle, respectively (Taylor et al. 2012).

While ignoring or underestimating the global impact of hematophagous flies, and, since the available control methods are expensive and poorly efficient, fly control is a part of livestock management which is most often neglected. Entomological and socio-economic studies are required to stress the importance of these insects, and, new methods are needed to complete their control. 
Whichever specific control method program is adopted, integrated management is a basic tool to be applied first (Saliba et al. 1976, Duvallet et al. 2017), such as general cleaning, elimination of water (mosquito larval habitat) (Fontenille et al. 2017), and manure and vegetation waste elimination (Muscidae larval habitat) to avoid offering culture media for insect larvae (Duvallet et al. 2017). However, killing adult insects remains necessary when facing a high seasonal peak of infestation. New methods are currently under development, to adapt insecticide-impregnated screens to attract and kill hematophagous flies (Desquesnes et al. 2019). Such tools might easily be adopted, once farmers could appreciate their potential benefit. A step before investing into a pest-control program is to evaluate the amount of money lost due to the concerned pest.

In the present study, we compared defense behaviour (tail flicks), haematocrit value, feed intake, growth performances and feed efficiency of two groups of similar growing beef cattle, kept free inside stables. One group was protected by a mosquito net and the other was kept in open-air conditions. To evidence the explanatory factors, insects' trappings were organised in the surrounding of the experimental site.

\section{Material And Methods}

\subsection{Study area and equipment}

This study was carried out in Kasetsart University, Kamphaeng Saen Campus, Nakhon Pathom Province, Thailand, between May 2016 and February 2017.

Two stables (A and B), $7 \times 12$ meters (84 sqm each), a third of each is covered by a roof, were delimited and separated 2 meters apart. Each of these stables was equipped with a feed trough and a water trough. In the middle of each stable, 1 meter under the roof, a device was installed to record temperature and hygrometry. Stable B was a normal open-air area. Stable A was entirely covered with a mosquito net (flyproof system), installed one meter apart from the fence and one meter apart from stable B (to avoid tearing off by the animals), including a mosquito roof at 2.5-3 meters high, and an additional entrancesas (airlock) to avoid introducing insects when coming in and out of the stable (Fig. 1). The inside of the airlock was equipped with a fan oriented towards the entrance, to avoid introducing flies when coming in and out, while bringing feed or removing cowpat. This fan was set up when using the entrance-sas.

Two fans were fixed in a high position, under the roof, inside each stable to blow the air towards the ground in order to lower the stable temperature, if necessary, to keep temperature as close as possible in both stables. As a rule, it was established that fans would be set-on in a stable when its temperature would be 2 degree Celcius over the temperature of the other stable. Temperatures of both stables were recorded 3 times a day, morning $(8: 00 \mathrm{am})$, noon (12:00) and evening $(5: 00 \mathrm{pm})$. If flies were found inside the mosquito net stable, they were cached and destroyed each day during morning and evening checks. In both stables, cowpat was removed daily, in the morning and the afternoon, to avoid any fly larval development.

\subsection{Animals housing, management and grouping}


Two experiments were carried out successively, applying the same protocol: Experiment 1, from 10th of May to 11th of July 2016, and Experiment 2, from 7th of September 2016 to 22nd of February 2017. For each experiment, twelve animals were selected out of a group of 30 weaned Kamphaeng Sean beef bullcalves (Sawanon et al. 2011), 6.5-12 months of age, issued from the Animal sciences breeding unit (Faculty of Agriculture, Kamphaeng Saen Campus, Kasetsart University). At first, all calves were kept free in an open-air 300 sqm park, and observed daily for 3 days by two technicians, to score the attractivity and the behaviour of the animals vis-à-vis the flies. Attractivity was estimated by the mean of 3 repeated counts of the total flies visible on one side of each animal, morning and evening, during 3 days; animals exhibiting extreme values were rejected. Reactivity/passivity was estimated by observation of defense movements; increasing intensity was scored from 0 to 4 at the same time as the insects counts. Animals exhibiting extreme reactivity (mean score $>3$ ) or extreme passivity (mean score $\leq 1$ ) were excluded from the experiment. Moreover, animals showing aggressive behaviour amongst each others or towards the manipulators were also rejected. Non-rejected animals were weighted before the selection was completed (using TAN Scale Model LP7110C-1T, Thailand). Age and weight were also considered, and, animals with extreme values were excluded. Remaining candidate animals were submitted to parasitological, serological and molecular blood tests for hemoparasites (see below). Only negative animals were kept for the experiment. Twelve bull-calves were selected as described above, and randomly split into two groups of 6 animals (Group A \& B), considering the age and the weight of the animals, to constitute two groups exhibiting as close as possible mean weight and mean age. These animals were treated against intestinal and blood parasites with Aben-15® (150 mg Albendazole; F.E. Pharma Company Limited $\AA$,

Thailand) and Ivomec ${ }^{\circledR}$ Plus (1\% Ivermectin and 10\% Clorsulon, Merial® Inc., USA). Animals were introduced into a pen ( $\mathrm{A}$ or $\mathrm{B}$ ) at the end of week 2 of each experiment (the first two weeks being during the preliminary period, when the 12 calves were kept together in open air).

\subsection{Follow-up of the animal's health}

From 30 days prior to the introduction of the cattle in the stables, and every 2 weeks during all the experiments, blood samples were collected to check as follows:

(i) the haematocrit value (pack cell volume, PCV\%) by capillary centrifugation method;

(ii) the presence of moving blood parasites (Trypanosoma, Filaria), by microscopic observation of the buffy coat under capillary centrifuge method (Woo 1969);

(iii) the presence of blood parasites (Anaplasma, Babesia, Trypanosoma, Theileria), by microscopic observation of Giemsa stained thin blood smears;

(iv) the presence of antibodies directed against T. evansi under Card Agglutination Test for Trypanosomes / T. evansi (CATT/ T. evansi) and Enzyme Linked Immuno-Sorbent Assay against Trypanosoma evansi (ELISA T. evansi) (Bajyana Songa et al. 1987, Desquesnes et al. 2009);

(v) the presence of Trypanozoon DNA by PCR (Chelex® prepared buffy coats) (Pruvot et al. 2013) using TBR primers with protocols previously published (Masiga et al. 1992, Pruvot et al. 2010). 


\subsection{Feed and feed-intake}

The concentrate diet and fresh para grass (Brachiaria mutica) were fed ad libitum, twice daily (at 07:00 a.m. and 05:00 p.m.). The ingredients and chemical compositions of the diets are shown in Table 1.

Table 1

Feed ingredients and chemical composition of concentrate feed and para grass

\begin{tabular}{|c|c|c|}
\hline Item & Concentrate & Para grass \\
\hline Molasses & 8.0 & \\
\hline Urea & 1.5 & \\
\hline Cassava chip & 30.0 & \\
\hline Solvent extracted palm kernel meal & 29.5 & \\
\hline Ground corn & 10.0 & \\
\hline Premix (Beef) $)^{1 /}$ & 20.0 & \\
\hline Sulfur & 0.5 & \\
\hline Salt & 0.1 & \\
\hline Di-calcium phosphate (Nuraphos-18) & 0.5 & \\
\hline \multicolumn{3}{|l|}{ Chemical composition } \\
\hline Dry matter DM (\%) & 89.3 & 23.1 \\
\hline Crude protein, CP (\%) & 16.5 & 6.7 \\
\hline Ether extract, EE (\%) & 4.2 & - \\
\hline Ash (\%) & 5.5 & 70.8 \\
\hline Neutral detergent fiber, NDF & - & 38.7 \\
\hline Acid detergent fiber, ADF & - & 5.4 \\
\hline Acid detergent lignin, $A D L$ & - & 11.1 \\
\hline $\mathrm{Ca}(\%)$ & 1.2 & - \\
\hline$P(\%)$ & 0.6 & - \\
\hline \multicolumn{3}{|c|}{ 1/Agromix beef No. 46: vitamin $A=2,160,000 \mathrm{IU}$, vitamin $B 3=100,000 \mathrm{IU}$, vitamin $E=5,000 \mathrm{IU}$, } \\
\hline \multicolumn{3}{|c|}{$\mathrm{Mn}=8.5 \mathrm{~g}, \mathrm{Zn}=6.4 \mathrm{~g}, \mathrm{Cu}=1.6 \mathrm{~g}, \mathrm{Mg}=16 \mathrm{~g}, \mathrm{Co}=320 \mathrm{mg}, \mathrm{I}=800 \mathrm{mg}, \mathrm{Se}=32 \mathrm{mg}$} \\
\hline
\end{tabular}

Weight of hay and concentrate delivered were daily recorded in the morning and the afternoon, as well as the weight of hay and concentrate remaining which were recorded in the morning of the next day; 
additionally, to evaluate a potential effect on the feed-ingestion rhythm of the animals, the food remaining before afternoon delivering was visually scored from 0 to 3 , and recorded for statistical analysis. At the beginning of the experiment, the first amounts of feed delivered were estimated based on the age and weight of the animals, but further on, this amount was increased, based on the amount ingested the day before, plus 2-3 kilos, so that a larger amount be presented anytime, and the feed be considered as delivered ad libitum. Animals had permanent free access to feed and clean drinking water.

\subsection{Insects trapping and identification}

As said in the indroduction, the most important Dipteran pests of livestock being the large size hematophagous Brachycera flies, Vavoua and Nzi traps, which are fairly adapted to such flies, were used (Laveissiere and Grebaut 1990, Mihok 2002, Bouyer et al. 2015). Once a week, in the pasture next to the stables (see the grass area in front of the stables on the upper picture, Fig. 1), two Vavoua traps and one Nzi trap were set up on a line at 60 meters apart, from 06:00 a.m. to 6:00 p.m., and the trapping-cages were installed, starting from 6:00 a.m., for 6 trapping-periods of 2 hours; cages were then collected at $8: 00,10: 00,12: 00,14: 00,16: 00$ and 18:00, to evaluate temporal distribution of flies along the day. Insects were identified using keys for Musca, tabanids and stomoxyine flies (Zumpt 1973, Tumrasvin and Shinonaga 1977, Burton 1978). The total number of insects trapped in a day was used as an evaluation of the insect density in the study area. In order to demonstrate the annoyance of flies on cattle, the number of insects trapped during each of the 6 trapping-periods were compared with the tail-counts recorded during the same periods, using a so-called "tail-pedometer" (a pedometer attached $20 \mathrm{~cm}$ from the basis of the tail); partial data will be reported here, but the detailed results of this study will be published elsewhere. Data analyses were made for "hematophagous flies" (Stomoxys spp. + Musca crassirostris + tabanids), and "common flies" (Musca spp. at the exception of M. crassirostris).

Once, in the midterm of Experiment 2, in order to evaluate the potential role of mosquitoes in the annoyance of cattle, a mosquito trapping session, using a CDC trap (Sriwichai et al. 2015) hanged 2.5m high in the center of stable B, was organized at night, from 6:00 p.m. on 21st November 2016 to 6:00 a.m. on 22nd November 2016; all insects trapped were identify and counted (Rattanarithiku et al. 2005, Rattanarithikul et al. 2005). In these experiments, insects' trapping and identification were recorded to help identifying the source of annoyance of cattle, but a complete study of fly seasonal variations and flies density linked with pedometer records will be detailed and published elsewhere.

\subsection{Defense movement evaluation in cattle}

Defense movements of cattle to fend off the insects include ear, tail, head, leg and skin movements (Warnes and Finlayson 1987); amongst them, tail-flicks proved to be a significant marker of biting fly annoyance (Raymond 1987, El-Laithy 2007, Gerry et al. 2007), reaching or passing 20 flicks per minute in case of medium to high fly density in cattle or buffaloes (El-Laithy 2013).

In order to count tail defense movements, Fitbit ${ }^{\circledR}$ pedometers were attached to the tail of the feeder cattle to collect "Pedometer Records" (PR). Fitbit zip ${ }^{\circledR}$ pedometers (manufactured by FitBit ${ }^{\circledR}$ inc., 199 Fremont street, San Francisco, CA 94105, USA) are developed for sports and activity monitoring; they are 
waterproof, powered by disposable batteries, and they continuously record movements ("steps"). Stepcounts can be downloaded, after Bluetooth synchronisation using an electronic device (smartphone or computer), from the FitBit $\circledast$ website. One pedometer was attached to the tail of each animal, at a distance of 15-20 cm from the basis of the tail, using a regular gaze-band first, covered by an elastic gaze-band and further on fixed with a sticky gaze-band (Neotape $2 \AA$ ). Pedometer records (PR) (so-called "steps" on the FitBit ${ }^{\circledR}$ website) of animals of both Groups were downloaded and saved three times a week, thanks to Fitbit ${ }^{\circledR}$ smartphone application, for further data analyse. Continuous diurnal and nocturnal data obtained from the electronic devices were recorded from 10th of May 2016 to 25th of January 2017. Nocturnal-PRs (from 6:00 pm to 6:00 am) and diurnal-PRs (from 6:00 am to 6:00 pm) were downloaded and recorded separately, to evaluate the relative impact of diurnal versus crepuscular and nocturnal insects. In this experiment, tail flicks were recorded to quantify the intensity of the annoyance during various periods of time, and thus to identify the source of annoyance of cattle, but a complete study and validation of this method, in links with insect trappings will be detailed and published elsewhere.

\subsection{Statistical analyses}

Statistical analyses were carried out with R3.6.0 (R-Development-core-team 2010), under R-studio environment. Means are indicated with $95 \%$ confidence intervals. Comparisons of mean weight and age of the animals of Group A and B at the beginning of Experiment 1 and 2, were carried out using t-test and the results were significant when $p$-value $<0.05$; the same method was used to compare feed intakes, dry matter intakes (DMIs) and feed conversion ratios (FCRs).

Construction of the $95 \%$ confidence interval for the difference in mean body weight between Group $A$ and Group B (small size groups, $\mathrm{n}<30$ ), was made using t-table at the appropriated degree of freedom, according to a method previously described (Lane 2019). As recommended, the following assumptions were checked: (i) there is homogeneity of the variances in the two groups; this was demonstrated by using Levene's test and it was accepted for $p$-values $>0.05$; (ii) the data of the two groups are normally distributed; this was checked by using Shapiro-Wilk test and it was accepted for $p$-values $>0.05$, and (iii) each value needs to be sampled independently from each other value (the case for individual animal weights in this study).

Comparison of Group A and B PCVs were made by two-way repeated measures ANOVA, and graphical representations were done using the package ggplot2 (Wickham 2016). Two periods of time were defined in Experiment 1, and 3 periods in Experiment 2, based on the graphical observation of "low" or "high" difference of the PCVs between the 2 groups.

For weight comparisons, graphical representations were done using the package ggplot2 (Wickham 2016), and, following recommendations previously published (Pinheiro and Bates 2000), for analyse, several models were tested, some using a Maximum Likelihood (ML) algorithm, but the one which seemed to best fit the data was the two-way repeated measures ANOVA (Zaiontz 2019). This method was applied to 4 different periods of time, based on the graphical observation of "high" or "low" insect 
densities recorded during in Experiment 1 and 2, before and at the beginning of the screens setting (period 1), and along 3 following periods, until the end of the experiments (periods $2-4$ ).

In both cases (PCVs and weights), as recommended, the following assumptions were checked: (i) homogeneity of the variances in the two groups (demonstrated by using Levene's test, and accepted for $p$-values > 0.05); (ii) normality of the data (checked by using Shapiro-Wilk test, and accepted for $p$-values $>0.05$ ), and (iii) individual data were collected independently from each other. The mean PCV and weight, in Group A and B were significantly different when the $p$-value $<0.05$ (http://www.realstatistics.com/anova-repeated-measures/one-between-subjects-factor-and-one-within-subjects-factor/ ) (Zaiontz 2019).

\section{Results}

The fly-proof pen was built as shown in Fig. 1, one meter aside the open-air pen. Temperature and relative humidity records were always very close in both pens. Temperature inside the fly-proof stable was sometime slightly lower than that of the open air, but never more than one degree Celcius or $5 \%$ relative humidity. As a consequence, inside fans were never used, and it was considered that the slight differences in temperature and relative humidity between the two pens did not interfere in the study.

\subsection{Animals and grouping}

Half of the 30 animals were rejected as described in material and methods. All animals tested proved to be negative to all hemoparasite-detection tests, and remained negative all through the experiments. Twelve calves 6.8-12.2 months of age were selected in Experiment 1 and 8.7-10.8 months of age in Experiment 2. Mean age of the animals was $314 \pm 34$ days in Experiment 1 and $299 \pm 15$ days in Experiment 2; there was no significant difference between the two groups (A / B) in both experiments (all $p$-values of t-test $>0.80$; Table 2). Mean weight of the animals was $281 \pm 22 \mathrm{Kg}$ in Experiment 1, and 223 $\pm 19 \mathrm{Kg}$ in Experiment 2; there was no significant difference between the two groups (A /B) in both experiments (Table 2) ( $p$-values of t-test $>0.75$ ). Experiment 1 was stopped after 9 weeks because of violent interactions between bulls, probably due to a too large age-range; for Experiment 2, more agesimilar calves were selected (8.7-10.8 months of age).

Experiment 1 lasted 9 weeks, from 10th of May to 11th of July 2016; animals were altogether during the first two weeks; animals of Group A entered the fly-proof device at the end of week 2, and spent 7 weeks under fly-proof conditions (week 3-9).

Experiment 2 lasted 24 weeks, from 7th of September 2016 to 22nd of February 2017; animals were altogether during the first two weeks; animals of Group A entered the fly-proof device at the end of week 2 , and spent 22 weeks under fly-proof conditions (week 3-24). 
Table 2

Mean age and weight of cattle Group A and B at the beginning of Experiment

1 and 2 , and $p$-values of t-tests

\begin{tabular}{|llll|}
\hline Experiment 1 & Group A & Group B & $p$-value (t-test) \\
\hline Mean body weight $\pm 95 \% \mathrm{Cl}(\mathrm{Kg})$ & $284 \pm 31$ & $278 \pm 45$ & 0.78 \\
\hline Mean age $\pm 95 \% \mathrm{Cl}$ (days) & $311 \pm 58$ & $318 \pm 59$ & 0.84 \\
\hline Experiment 2 & & & \\
\hline Mean body weight $\pm 95 \% \mathrm{Cl}(\mathrm{Kg})$ & $223 \pm 38$ & $224 \pm 26$ & 0.93 \\
\hline Mean age $\pm 95 \% \mathrm{Cl}$ (days) & $300 \pm 24$ & $299 \pm 27$ & 0.94 \\
\hline
\end{tabular}

\subsection{Insect trapping and identification}

On average in Experiment 1, hematophagous flies (Stomoxys spp. + M. crassirostris + tabanids) were in close proportions with common flies (Musca spp., at the exception of M. crassirostris), with $49.3 \%$ of the total catches), but they represented 2/3rd of the total flies in Experiment 2 (63.1\%). On average the hematophagous flies trapped were dominated by Stomoxys spp. and M. crassirostris, a non-biting but obligatory blood-sucking fly, highly prevalent in this area (Desquesnes et al. 2018), while tabanids remained in low numbers. Insect trapping results along Experiment 1 and 2 are presented on Fig. 2a (Experiment 1, 9 weeks, from 10th of May to 11th of July 2016) and Fig. 2b (Experiment 2, 24 weeks, from 7th of September 2016 to 22nd of February 2017). The daily total insects trapped in the 3 traps was comprised between 547 and 2,933 in Experiment 1, while it was between 104 and 3,676 in Experiment 2.

A night trapping using CDC-trap showed a huge activity of mosquitoes, with a total of 2,741 mosquitoes caught during a single night on 21st-22nd of November 2016. Ninety percent of the mosquitoes were trapped between 06:00 p.m. and 12:00 p.m., indicating a huge activity in the late evening-early night. Apart from mosquitoes, no other significant number of pest insects were caught at night.

\subsection{Comparison of mean hematocrit values}

Hematocrit values (quantified every two weeks by the pack cell volume (PCV)) were considered as good indicators of blood despoliation caused by hematophagous flies. The PCVs of the two groups of animals evolved as indicated in Fig. 3. Mean PCVs of Group A and B established at the beginning of the experiments (week 1 ) were not significantly different in Experiments 1 ( $p$-value 0.17 ) and 2 ( $p$-value 0.94 ). The mean hematocrit values of Group B became significantly lower than that of Group A in both experiments.

In Experiment 1 (Fig. 3a and Table 3a), based on a "low" or "high" difference of mean PCV in the two Group A and B observed on Fig. 3a, periods were defined as Period 1: weeks 1-4, with no or low difference between Group A and B, and Period 2: weeks 5-9, with higher difference between Group A and B. For Period 1, results of the two-way repeated measures ANOVA between Group A and B provided a $p$ value of 0.175 , leading to a non-significant difference between Group $A$ and $B$ at the beginning and early 
stage of Experiment 1. Conversely, results of the two-way repeated measures ANOVA between Group A and B provided a $p$-value of 0.001 for Period 2, which means a significant difference between Group A and $\mathrm{B}$.

In Experiment 2, PCV data are represented in Fig. 3b and Table 3b; the Experiment was split into 3 periods of low, high and medium differences between the 2 groups: Period 1: weeks 1-5 (before and just after Group A entered the fly-proof stable); Period 2: weeks 6-14 (high difference), and Period 3: weeks 15-24 (medium difference between the two groups). For the first period, results of the two-way repeated measures ANOVA provided a $p$-value of 0.452 , which showed non-significant difference between Group A and B. However, results of the two-way repeated measures ANOVA between Group A and B provided $p$ values of 0.000 and 0.006 for the second and third period, which demonstrated highly significant differences between PCVs of Group A and B.

Overall, these results demonstrated that flies exposure significantly affected the PCV of the exposed animals, with mean PCV values 3-4\% below the control group. In Experiment 2, the animals tended to recover their normal value when the fly density decreased. However, in the two experiments, PCVs of test groups never reached the level of control groups before the end of the experiments (final PCV of Group B $30.5 \%$ versus 33.2\% in Group A, in Experiment 1, and, 30.2\% versus 32.8\% in Experiment 2).

Table 3

a: Results of two-way repeated measures ANOVA on PCVs for Experiment 1

\begin{tabular}{|llll|}
\hline Source of variation & F statistic & F critical & p-values \\
\hline \multicolumn{2}{|l}{ Time (Week 1-4 for first period) } & & \\
\hline Groups (A vs. B) & 1.981 & 4.351 & 0.175 \\
\hline \multicolumn{3}{|l}{ Time (Week 5-9 for second period) } & \\
\hline Groups (A vs. B) & 13.143 & 4.171 & $\mathbf{0 . 0 0 1}$ \\
\hline
\end{tabular}

Table 3

b: Results of two-way repeated measures ANOVA on PCVs for Experiment 2

\begin{tabular}{|llll|}
\hline Source of Variation & F statistic & F critical & p-values \\
\hline \multicolumn{3}{|l}{ Time (Week 1-5 for the first period) } & \\
\hline Groups (A vs. B) & 0.580 & 4.171 & 0.452 \\
\hline Time (Week 6-14 for the second period) & \\
\hline Groups (A vs. B) & 15.468 & 4.085 & 0.000 \\
\hline Time (Week 15-24 for the third period) & \\
\hline Groups (A vs. B) & 8.225 & 4.034 & 0.006 \\
\hline
\end{tabular}

Page 12/26 


\subsection{Comparison of mean cattle weight}

The mean weight of cattle in Group A and B, and the number of hematophagous flies trapped per week during Experiment 1 (from 10/05/16 to 11/07/16) are reported in Fig. 4a. At the beginning of the experiment, the two groups showed similar mean weight (Group A: $284 \pm 31 \mathrm{Kg}$, Group B: $278 \pm 45 \mathrm{Kg}$ ), but when the insects density increased (weeks 3-5), the difference between the mean weights of Group $A$ and $B$ increased to reach a maximum of $12.5 \pm 4.9 \mathrm{Kg}$ in favor of Group $A$ on week 5 , at the peak of insect density $(2,149$ hematophagous insects trapped in one day).

Result of the two-way repeated measures ANOVA on cattle weight in Experiment 1 are presented in Table 4a. Week 1 and 2 were grouped for the first period (before Group A entered the fly-proof stable), week 3 to 5 for the second period (high fly density), week 6 to 7 for the third period (low fly density) and weeks 8 to 9 for the fourth period (high fly density). Results of two-way repeated measures ANOVA between Group A and $B$ provided a $p$-value $>0.05$ for the 4 periods; consequently, no significant difference could be demonstrated, although the mean weight of Group B was consistently and increasingly below that of Group A.

Table 4

a: Results of two-way repeated measures ANOVA on weight for Experiment 1

\begin{tabular}{|c|c|c|c|}
\hline Source of Variation & F statistic & F critical & $p$-values \\
\hline \multicolumn{4}{|c|}{ Time (Week $1-2$ for the first period) } \\
\hline Groups (A vs. B) & 0.161 & 4.351 & 0.693 \\
\hline \multicolumn{4}{|c|}{ Time (Week 3-5 for the second period) } \\
\hline Groups (A vs. B) & 0.333 & 4.171 & 0.568 \\
\hline \multicolumn{4}{|c|}{ Time (Week 6-7 for the third period) } \\
\hline Groups (A vs. B) & 0.413 & 4.351 & 0.528 \\
\hline \multicolumn{4}{|c|}{ Time (Week 8-9 for the fourth period) } \\
\hline Groups (A vs. B) & 0.566 & 4.351 & 0.461 \\
\hline
\end{tabular}

Experiment 2: the mean weight of cattle in Group A and B, and the number of hematophagous flies trapped per week during Experiment 2 (from 07/09/16 to 22/02/17) are reported in Fig. 4b. At the beginning of the experiment, the two groups showed similar mean weight (Group A: $223 \pm 38 \mathrm{Kg}$, Group B: $224 \pm 26 \mathrm{Kg}$ ), but, when the insects density increased (weeks 3-24), the average growths in Group A and $B$ departed, and became significantly different. The mean loss of LBWG of group B was around 1.8 $\mathrm{Kg} /$ week [0.6-3.1], variable from a period to another, reaching $36.3 \mathrm{Kg}$ over a period of 15 weeks (week 3-17), up to $40.0 \mathrm{Kg}$ over a period of 20 weeks (W3-24), which can be summarized in a mean loss of LBWG of $8.0 \pm 1.5 \mathrm{Kg} /$ month [2.7;13.3]. On week 24, the loss of LBWG reached $10.6 \%$ of the mean LBW of animals in Group B (the mean weight of animals in Group B being $378 \mathrm{Kg}$ at that time, versus $418 \mathrm{Kg}$ in 
Group A). Results of the two-way repeated measures ANOVA on cattle weight in Experiment 2 are summarized in Table 4b. Four periods were defined as follows: week 1-3 were grouped for the first period (prior to and just after group A entered the fly-proof stable), weeks 4-13 were grouped for the second period (Group B was exposed to high fly density), week 14-20 for the third period (low fly density) and weeks 21-24 for the fourth period (relapse of high fly density).

The two-way repeated measures ANOVA on cattle weight between Group A and B provided a $p$-value > 0.05 (0.971) for the first period; consequently, no significant difference could be demonstrated between the mean weight of Group A and B at the beginning of the Experiment. Conversely, results of the two-way repeated measures ANOVA between Group A and B provided $p$-values $<0.05$ for the 3 following periods, during which Group $B$ alone was exposed to fly activity, demonstrating a significant difference between the mean weight of Group A and B.

At the 22nd week of experiment, after the second peak of hematophagous flies, the difference between the mean weights of Group A and B was $40 \pm 5.5 \mathrm{Kg}$ in favor of Group A.

Table 4

b: Results of two-way repeated measures ANOVA on weight for Experiment 2

\begin{tabular}{|c|c|c|c|}
\hline Source of Variation & F statistic & F critical & $p$-values \\
\hline \multicolumn{4}{|c|}{ Time (Week $1-3$ for the first period) } \\
\hline Groups (A vs. B) & 0.001 & 4.171 & 0.971 \\
\hline \multicolumn{4}{|c|}{ Time (Week $4-13$ for the second period) } \\
\hline Groups (A vs. B) & 7.012 & 3.963 & 0.009 \\
\hline \multicolumn{4}{|c|}{ Time (Week $14-20$ for the third period) } \\
\hline Groups (A vs. B) & 12.560 & 3.978 & 0.001 \\
\hline \multicolumn{4}{|c|}{ Time (Week 21-24 for the fourth period) } \\
\hline Groups (A vs. B) & 7.880 & 4.085 & 0.008 \\
\hline
\end{tabular}

\subsection{Feed conversion ratio}

Weights of concentrate-, hay- and dry matter-intakes (DMIs) in Experiment 1 and 2, are summarized in Table 5. Feed intakes were not significantly different in Group A and B in both experiment (all $p$-values > 0.45). The mean weekly feed conversion ratios (FCRs) were calculated as the rate of weekly dry matter intake (DMI) and weekly weight gain. In Experiment 1, the mean FCR of Group B was greater than that of Group A, but not significantly. However, the mean FCRs were significantly different in Experiment 2 ( $p<$ $0.01)$. On average, in Group A (protected from flies) every $\mathrm{Kg}$ of weight-gain needed $4.97 \pm 0.50 \mathrm{Kg}$ of DMI, while, under fly exposure, in Group B, $6.62 \pm 1.19 \mathrm{Kg}$ of DMI were needed. Consequently, the feed required 
to obtain $1 \mathrm{Kg}$ of weight gain was increased by $1.65 \mathrm{Kg}$ of DMI for animals exposed to flies, which represented a total need of $133 \%$ of the DMI needed in control group.

As concerns the rhythm of feed ingestion, remaining feed scores in the afternoon (indicating the amount of food not ingested daytime) were slightly higher in Group B than Group A for concentrate (1.57 \pm 0.16 > $1.53 \pm 0.15)$, as well as for hay $(1.38 \pm 0.16>1.32 \pm 0.16)$, however these differences were not statistically significant in Experiment 1. In Experiment 2, afternoon remaining feed scores of Group B were slightly lower than Group A for concentrate $(1.59 \pm 0.08<1.62 \pm 0.08)$, but higher for hay $(1.46 \pm 0.09>1.40 \pm$ 0.09); here again, differences were not statistically significant, indicating that, overall the experiments, the fly pressure on Group B was not high enough to divert the animals from feeding daytime.

Table 5

Summary of t-test.statistical analysis on FCRs in Group A and B in Experiment 1 and 2

\begin{tabular}{|c|c|c|c|c|}
\hline Experiment 1 ( 9 weeks) & Group A & Group B & diff A-B & $p$-values \\
\hline Total concentrate ingested $(\mathrm{Kg})$ & 1422 & 1420 & 2 & 0.97 \\
\hline Total grass ingested $(\mathrm{Kg})$ & 1043 & 1045 & -2 & 0.96 \\
\hline Total dry matter intake (DMI) Kg & 2860 & 2875 & -15 & 0.95 \\
\hline Mean weekly FCR & 5.65 & 6.36 & -0.71 & 0.13 \\
\hline Experiment 2 (24 weeks) & Group A & Group B & diff A-B & p-values \\
\hline Total concentrate ingested (Kg) & 5294 & 5364 & -70 & 0.49 \\
\hline Total grass ingested $(\mathrm{Kg})$ & 4694 & 4704 & -10 & 0.95 \\
\hline Total dry matter intake (DMI) Kg & 6453 & 6526 & -73 & 0.89 \\
\hline Mean weekly FCR & 4.97 & 6.62 & -1.65 & 0.01 \\
\hline
\end{tabular}

Overall Experiment 2, on a daily basis, tail pedometer records (TPRs) in Group A revealed very low frequency of tail flicks or "steps", always below 200 beats per 2 hours, day-time, with maximums at the time of feed delivery, early morning and late afternoon, and very low frequency recorded at night, generally below 50 beats per 2 hours. Between September 2016 and February 2017, the mean daily TPRs recorded in Group A remained below 800 (Table 6), with a mean number of diurnal and nocturnal beats of $573 \pm 69$ and $164 \pm 24$ respectively. These records may be considered as the basic activity of cattle in the absence of fly annoyance, during this period of time and under these feeder-cattle breeding conditions. The rate of nocturnal "steps" was low and stable, representing around $22 \% \pm 1 \%$ of the total TPRs.

All data from Group B were highly significantly different from those of Group A $(p<0.05)$; thus, TPRs generally revealed high frequency of tail flicks in the morning (frequently above 1,000 steps/2 hours) and up to 2,500 steps/ 2 hours or more in the evenings, with a strong correlation between "steps" recorded and 
peaks of hematophagous fly density, in links with the bimodal activity of the insects during this season (data not shown). Overall in Group B, the mean daily diurnal TPRs reached 5,333 $\pm 2,044$ beats ( 9 times higer than that of Group A), with a high and variable rate of nocturnal TPRs, ranging from 15 to $74 \%$, and averaging $40 \% \pm 15 \%$ (Table 6), which was 22 times higher than that of Group A. To evaluate the number of beats due to insects, we deduced TPRs of Group A (beats due to the normal basic activity of cattle) from TPRs of Group B (beats due to the basic activity of cattle + defence movements to the insects) (Table 6, last column); which lead to mean additional numbers of beats due to insects of $3,382 \pm 1,855$ at night and 4,761 $\pm 2,106$, day-time. These data suggest a significant cattle annoyance at night (on average $40 \%$ of the total defence movements of cattle per 24 hours). Insects trapped during one night-trapping using CDC trap hanged in the pen of Group B, in November 2016, were 3,120 specimens, principally represented by mosquitoes, with 2,741 specimens ( $88 \%)$, while very few other dipteran pests were trapped: 16 Stomoxys spp. (0.5\%) and 24 common flies (0.8\%). Other insects trapped were principally small coleopterans (6.8\%) and lepidopterans (3.54\%), and very low numbers of hymenopterans $(0.4 \%)$ and hemipterans $(0.1 \%)$. From these scores, it was strongly hypothesized that mosquitoes were responsible of the high numbers of tail-pedometer beats recorded at night in Group B. A more detailed analysis of these data and results will be presented elsewhere.

Table 6

Mean diurnal and nocturnal TPRs of Group A and B in Experiment 2.

\begin{tabular}{|lllll|}
\hline tail pedometer records (PR) & Group A & Group B & p-value (t-test) & Group B-Group A \\
\hline Mean diurnal TPRs $\pm 95 \% \mathrm{Cl}$ & $573 \pm 69$ & $5,333 \pm 2,044$ & 0.002 & $4,761 \pm 2,106$ \\
\hline Mean nocturnal TPRs $\pm 95 \% \mathrm{Cl}$ & $163 \pm 24$ & $3,545 \pm 1,856$ & 0.008 & $3,382 \pm 1,855$ \\
\hline Mean 24 hours TPRs $\pm 95 \% \mathrm{Cl}$ & $736 \pm 91$ & $8,879 \pm 3,545$ & 0.003 & $8,143 \pm 3,068$ \\
\hline Percentage of diurnal TPRs & $78 \% \pm 1 \%$ & $61 \% \pm 14 \%$ & 0.019 & $60 \% \pm 15 \%$ * \\
\hline Percentage of nocturnal TPRs & $22 \% \pm 1 \%$ & $39 \% \pm 14 \%$ & 0.019 & $40 \% \pm 15 \%$ * \\
\hline * established on the mean monthly results & & & \\
\hline
\end{tabular}

\section{Discussion/conclusion}

Our results suggest a significant negative impact of hematophagous flies, on cattle annoyance, health and growth.

\subsection{Discussion}

Fly densities, peculiarly hematophagous flies, were quite variable during the two experiments; however, in both cases, they exhibited two serial peaks, which induced fluctuations in the impact-parameters recorded. Overall, fly densities were considered as high ( $>400$ hematophagous flies /trap/day) during half of Experiment 1 (weeks 4-5 and 8-9) and Experiment 2 (weeks 4-14 and 18-22); they were lower in 
other periods. The impact recorded on cattle might then be limited to a half of the experimental periods, thus, higher impact may be expected in case of higher or more constant fly density.

Direct impact of flies on cattle behavior was obvious from the tail-pedometer records (TPRs), with, on average, more than 12 times more "steps" recorded in Group B (mean 24 hours TPR 8,879 $\pm 3,545$ ) versus Group A (736 \pm 91$)$ in Experiment 2; however, a part of these defense movements were due to nocturnal insects as discussed below, when splitting diurnal and nocturnal TPRs.

Beside the tail flicks, the feed intake could possibly be affected by the flies' annoyance, however, in the conditions of our experiments, the feed intake was not significantly different in the two groups, both in terms of feed-ingestion rhythm and total weight of feed and dry matter ingested. However, the feed conversion ratio (DMI/weight gain), was higher in the exposed group, with a strong statistical significance in Experiment 2 (Group B needed 133\% of the DMI needed by Group A for a similar weight gain), allowing to conclude to a decreased feed-efficiency induced by the insect's annoyance. Similar results had been previously obtained in 4 ways mixed cattle feedlots (mean weight of $272 \mathrm{Kg}$ at the beginning of the experiment) under the pressure of natural Stomoxys spp. population in Nebraska (Campbell et al. 1987).

Due to blood cells ingestion, hematophagous flies are expected to impact the hematocrit of exposed cattle; indeed, a significant impact was recorded in groups B, especially in Experiment 2 (lasting longer than Experiment 1), where the mean PCV of exposed animals reached a value 3-4\% below that of the control group, synchronized with the high fly density periods (in Experiments 1 and 2), and showing an immediate direct effect of hematophagous flies on this cattle health blood parameter.

The live body weight gain (LBWG) of cattle was also impacted by high fly densities, as shown by the highly significant results obtained when comparing LBWG of groups A and B. In Experiment 2, the mean loss of LBWG of group B was of $8.0 \pm 1.5 \mathrm{Kg} /$ month [2.7;13.3]. This loss being recorded under a "medium" fly pressure (apparent density per trap $<500$ in both experiments), greater losses can be expected under higher fly pressure.

A direct link between LBWG and flies density is not easy to draw, since other parameters are impacting weight gains, such as compensatory growing after a period of reduced growing, habituation of the cattle to high fly density after a period of exposure, climatic conditions, interactions between cattle in a group etc. However, the global impact of flies on the weight gain measured in this study is calling for a serious attention in terms of loss of incomes. In order to use a universal unit, we calculated the shortfall of the weight gain on feeder cattle, taking as a model the results of Experiment 2. In this experiment, within 20 weeks of exposure to flies (weeks 3-22), the loss of LBWG of Group B reached $40.0 \pm 5.5 \mathrm{Kg}$. This loss of LBWG amounted $10.6 \%$ of the LBW of exposed cattle, lost within five months, during their main growing period (10-15 months of age). When considering the current value of KPS beef LBW in Thailand (estimated around $125 \mathrm{THB} / \mathrm{Kg}$ or $4 \mathrm{USD} / \mathrm{Kg}$ in 2019), a feedlot of a 100 calves would amount a total loss of around 4 tons of LBW, for a value of $\$ 16.000$. 
In a study carried out in the US, based on the cattle inventories and average prices for 2005-2009, and median monthly infestation levels, national losses were estimated to be $\$ 360$ million for dairy cattle, \$358 million for cow-calf herds, \$1,268 million for pastured cattle, and \$226 million for cattle on feed, for a total impact to U.S. cattle industries of $\$ 2,211$ million per year. We would hardly extrapolate our results to a whole country, but, depending on farming conditions, the economic impact of flies might be very serious, and could be roughly estimated around $10 \%$ of the cattle LBW, lost during a five months period of medium to high flies density. Our results are quite consistent with previous estimations made in the US (West Central Nebraska), in which, on average, losses of weight gain recorded in grazing yearling cattle exposed to Stomoxys calcitrans were estimated around $0.2 \mathrm{Kg} /$ day (Campbell et al. 2001); extrapolated to a period of five month, the loss would amount to a total of $30 \mathrm{Kg}$ of LBW/head. Our results (a total loss of $40 \mathrm{Kg}$ LBW/head) being obtained only over a 5 months period, it is possible that the longer observation made by Campbell et al somehow "buffered" the effects of flies over time, or, and additional effect of the mosquitoes was registered in our case. In another study, in free pasturing cattle, the mean additional weight gain in insecticide treated animals was of $8.2 \mathrm{Kg}$ per month, which represent $41 \mathrm{Kg}$ for 5 months (Bruce and Decker 1951a, Bruce and Decker 1951b); results, again, very close to ours.

The present study was designed to measure the impact of hematophagous flies on cattle. However, due to the fly-proof system set-up, our device automatically included a protection against mosquitoes, and the impact measured on cattle kept in open-air also included the effects of flies and mosquitoes on cattle. Evaluating the potential relative role of mosquitoes on cattle, based on the total numbers and rates of diurnal versus nocturnal tail-pedometer records in Experiment 2, the cattle behavior was seriously impacted by insects at night, to which could be attributed, on average, 3,545 beats/night, representing $40 \%$ of the additional beats recorded per 24 hours in exposed animals, versus control (Table 6). Whether there is a direct link between the number of tail flicks (or TPRs) and the loss of LBWG is a difficult point to conclude on. Responsibility of the losses measured in this study may be shared between hematophagous flies day-time and mosquitoes night-time, but the relative impact of flies and mosquitoes would be difficult to establish, unless a specific experimental study be designed for that purpose. Most of the authors use to consider mosquitoes as negligible pests for livestock, as said by Hill : « The feeding adults will take some blood and cause some irritation, but not usually a great deal » (Hill 1997). In fact, annoyance and abundance of mosquitoes are certainly under-estimated because they occur at night, after the end of working-hours. The potential impact of mosquitoes on livestock should be reconsidered in the light of our results. As an example, in dairy cattle farms in Thailand, where farmer's habitations are very close to cattle pens and stable, animals' owners very often complain about huge mosquito annoyance for themselves and their cattle. A topic on which limited investigations were carried out so far. On that aspect, the use of tail pedometers proved to be very useful, by providing continuous nocturnal and diurnal data that can easily be split to analyse relative diurnal and nocturnal pest attacks.

\subsection{Conclusion}

These experiments allowed to demonstrate the highly significant impact of flying insects on cattle, including: (i) a high loss of energy evidenced by tail flicks pedometer-records, showing, day-time, 12 times 
more tail flicks in cattle exposed to flies, (ii) a red cells depletion evidenced by a significantly decreased PCV in exposed animals, $3-4 \%$ below the control group, (iii) a decreased cost-benefit on the feed, due to significantly increased by $33 \%$ of feed conversion ratio (FCR) in exposed cattle, (iv) a significant loss averaging $10-11 \%$ of LBWG in KPS beef feeder cattle 10-15 month of age, accounting for a money loss of $\$ 160$ within 5 months, and $(v)$ a non-negligible potential impact of mosquitoes on cattle, evidenced by an important increase of tail flick defense movements at night (averaging 22 times more TPRs than nonexposed animals), requiring further evaluation.

Overall the 5-month study in Experiment 2, diurnal TPRs represented $60 \%$ of the total TPRs in exposed animals, suggesting a predominant impact of hematophagous flies on cattle, however, leaving room for a potential role of mosquitoes in total losses. The total loss of LBWG registered in this study was equivalent to $10-11 \%$ of the cattle LBW, lost within a season of insect activity; a part of this money could fruitfully be invested in fly control, providing control methods are safe and cost-efficient.

\section{Declarations}

\section{Acknowledgements}

The authors wish to thank all farmers from Nong Pho area who friendly accepted to host our experiments and observations. The authors would like to thank the Department of Entomology, Faculty of Agriculture at Kamphaeng Saen and the Faculty of Veterinary Medicine, Kasetsart University, Thailand, for support and laboratory expenses and facilities access. This work was funded by the Centre de Coopération Internationale en Recherche Agronomique pour le Développement (CIRAD) and the French National Research Agency [Agence Nationale de la Recherche; ANR], under the « FlyScreen' project » (ANR-15CE35-0003). This work was partially supported by the Center for Advanced Studies for Agriculture and Food, Institute for Advanced Studies, Kasetsart University Under the Higher Education Research Promotion and National Research University Project of Thailand, Office of the Higher Education Commission, Ministry of Education, Thailand.

\section{Statement of Animal Rights}

The handling of animals was performed in strict accordance with good animal practice guidelines and the study was approved by the $\mathrm{KU}$ ethics committee

\section{Disclosure of interest conflicts}

The authors declare no conflict of interest.

\section{Authors contributions:}

PB, RM, SJ and MD conceived and designed study

$\mathrm{PB}, \mathrm{AN}, \mathrm{SO}, \mathrm{CF}, \mathrm{PC}, \mathrm{AL} \& \mathrm{MD}$ performed the research 
AN, CF, KT, A, ST \& MD analyzed data

MD contributed new methods or models

$\mathrm{PB}, \mathrm{AN}, \mathrm{KT}, \mathrm{ST} \& \mathrm{MD}$ wrote the paper

\section{9) Data availability statement :}

The datasets generated during and/or analysed during the current study are available from the corresponding author on reasonable request.

\section{References}

1. Bajyana Songa, E., Hamers-Casterman, C., Hamers, R., Pholpark, M., Pholpark, S., Leidl, K., Tangchaitrong, S., Chaichanopoonpol, I., Vitoorakool, C. \& Thirapatsakum, T. (1987) The use of the card agglutination test (Testryp CATT) for the detection of $T$. evansi infection: a comparison with other trypanosomiasis diagnostic tests under field conditions in Thailand. Annales de la Société Belge de Médecine Tropicale 67(2), 137-148.

2. Baldacchino, F., Desquesnes, M., Mihok, S., Foil, L.D., Duvallet, G. \& Jittapalapong, S. (2014) Tabanids: neglected subjects of research, but important vectors of disease agents! Infect Genet Evol 28, 596-615. DOI: 10.1016/j.meegid.2014.03.029.

3. Baldacchino, F., Muenworn, V., Desquesnes, M., Desoli, F., Charoenviriyaphap, T. \& Duvallet, G. (2013) Transmission of pathogens by Stomoxys flies (Diptera, Muscidae): a review. Parasite 20, 26. DOI: 10.1051/parasite/2013026.

4. Bauer, B., Gitau, D., Oloo, F.P. \& Karanja, S.M. (2006) Evaluation of a preliminary title to protect zerograzed dairy cattle with insecticide-treated mosquito netting in western Kenya. Trop Anim Health Prod 38(1), 29-34.

5. Bouyer, J., Desquesnes, M., Yoni, W., Chamisa, A. \& Guerrini, L. (2015) Attracting and trapping insect vectors. GeosAf 2, 23pp.

6. Bruce, W.N. \& Decker, G.C.(1951a) Control of horseflies on cattle. Natural history survey division; Biological Notes No. 24. R a Education. Urbana, Illinois, Department of registration and education. 24: 1-8.

7. Bruce, W.N. \& Decker, G.C. (1951b) Tabanid control on dairy and beef cattle with synergized pyrethrins. J econ Entomol 44, 154-159.

8. Burton, J. (1978) Tabanini of Thailand above the Isthmus of Kra (Diptera: Tabanidae). Entomological Reprint Specialists, Los Angeles, USA, 165.

9. Byford, R., Craig, M. \& Crosby, B. (1992) A review of ectoparasites and their effect on cattle production. J. Anim. Sci. 70, 597-602.

10. Campbell, J.B., Berry, I. L. , Boxler, D.J., Davis, R.L., Clanton, D.C. \& Deutscher, G.H. (1987) Effects of stable flies (Diptera: Muscidae) on weight gain and feed efficiency of feedlot cattle. J. Econ. Entomol. 
(80), 177-119.

11. Campbell, J.B., Skoda, S.R., Berkebile, D.R., Boxler, D.J., Thomas, G.D., Adams, D.C. \& Davis, R. (2001) Effects of stable flies (Diptera: Muscidae) on weight gains of grazing yearling cattle. J Econ Entomol 94(3), 780-783.

12. Desquesnes, M. (2004) Livestock trypanosomoses and their vectors in Latin America. CIRAD-EMVT publication, OIE, Paris, ISBN 92-9044-634-X,

13. Desquesnes, M., Bouhsira, E., Chalermwong, P., Drosne, L., Duvallet, G., Franc, M., Gimonneau, G., Grimaud, Y., Guillet, P., Himeidan, Y., Jacquiet, P., Jittapalapong, S., Karanja, W., Liénard, E., Onju, S., Ouma, J., Rayaisse, J.-B., Masmeatathip, R., Salou, E., Shah, V., Shukri, S. \& Thaisungnoen, K. (2019) Insecticide-impregnated screens used under « multi target method» for hematophagous fly control in cattle: a proof of concept. ECVD accepted.

14. Desquesnes, M., Kamyingkird, K., Pruvot, M., Kengradomkij, C., Bossard, G., Sarataphan, N. \& Jittapalapong, S. (2009) Antibody-ELISA for Trypanosoma evansi: application in a serological survey of dairy cattle, Thailand, and validation of a locally produced antigen. Prev Vet Med 90(3-4), 233-241. DOI: 10.1016/j.prevetmed.2009.04.011.

15. Desquesnes, M., Onju, S., Chalermwong, P., Jittapalapong, S. \& Masmeatathip, R. (2018) A review and illustrated description of Musca crassirostris, one of the most neglected haematophagous livestock flies. Medical and Veterinary Entomology, 15. DOI: 10.1111/mve.12339.

16. Duvallet, G. (2017) Généralités sur les diptères (Diptera). Entomologie médicale et vétérinaire. F e R Duvallet and I Edition, Marseille, Versailles: 165-179.

17. Duvallet, G., Baldacchino, F. \& Desquesnes, M. (2017) Stomoxyini (Diptera: Muscidae: Muscinae). Entomologie Médicale et vétérinaire. F R Duvallet, IRD Editions. Marseille, Versailles: 391-403.

18. El-Laithy, S. (2007) Effect of Fly Annoyance on Dairy Cattle Behaviour and Milk Production. Benha Vet Med J 18(2), 1-12.

19. El-Laithy, S. (2013) Effect of Fly Annoyance on Buffalo Behavior and Milk Production. Global Veterinaria 10(4), 472-479.

20. Fontenille, D., Paupy, C. \& Failloux, A.B. (2017) Culicinae (Diptera: Culicidae). Entomologie Médicale et vétérinaire. F R Duvallet, IRD Editions. Marseille, Versailles: 243-294.

21. Gerry, A., Peterson, N. \& Mullens, B. (2007) Predicting and Controlling Stable Flies on California Dairies. UCANR 8258, 1-11.

22. Hill, D. (1997) The Economic Importance of Insects. Chapmann \& Hall, 98-102.

23. Hogsette, J.A., Nalli, A. \& Foil, L.D. (2008) Evaluation of different insecticides and fabric types for development of treated targets for stable fly (Diptera: Muscidae) control. J Econ Entomol 101(3), 1034-1038.

24. Lane, D.(2019) Part 11: Differences between means standard. Online Statistics Education: An Interactive Multimedia Course of Study (http://onlinestatbook.com/). R University. Houston, Texas, Rice University. 
25. Laveissiere, C. \& Grebaut, P. (1990) [The trapping of tsetse flies (Diptera: Glossinidae). Improvement of a model: the Vavoua trap]. Trop Med Parasitol 41(2), 185-192.

26. Masiga, D.K., Smyth, A.J., Hayes, P., Bromidge, T.J. \& Gibson, W.C. (1992) Sensitive detection of trypanosomes in tsetse flies by DNA amplification. International Journal of Parasitology 22, 909-918.

27. Mihok, S. (2002) The development of a multipurpose trap (the Nzi) for tsetse and other biting flies. Bull Entomol Res 92(5), 385-403. DOI: 10.1079/BER2002186.

28. Minár, J., Kostenko, L. \& Ríha, J. (1987) Decrease in the milk yield in cows casued by dipterous bloodsucking insects and the protection of heifers with Oxamate, a repellent, in the area of Leningrad. Vet Med (Praha) 32(6), 355-363.

29. Patton, W. (1922) Notes on the species of the genus Musca, Linneatus- Part I. Bulletin of Entomological Research 12(4), 411-426.

30. Pinheiro, J. \& Bates, D. (2000) Mixed-effects models in S and S-PLUS; . New-York,

31. Pruvot, M., Kamyingkird, K., Desquesnes, M., Sarataphan, N. \& Jittapalapong, S. (2010) A comparison of six primer sets for detection of Trypanosoma evansi by polymerase chain reaction in rodents and Thai livestock. Vet Parasitol 171(3-4), 185-193. DOI: 10.1016/j.vetpar.2010.04.001.

32. Pruvot, M., Kamyingkird, K., Desquesnes, M., Sarataphan, N. \& Jittapalapong, S. (2013) The effect of the DNA preparation method on the sensitivity of PCR for the detection of Trypanosoma evansi in rodents and implications for epidemiological surveillance efforts. Vet Parasitol 191(3-4), 203-208. DOI: 10.1016/j.vetpar.2012.09.010.

33. R-Development-core-team (2010) R: A Language and Environment for Statistical Computing. . Vienna, Austria, http://www.Rproject.org.

34. Rattanarithiku, I.R., Harrisson, B., Panthusin, P. \& Coleman, R. (2005) Illustrated keys to the mosquitoes of thailand; I. Background ; geographic distribution ; lists of genera, subgenera, and species ; and a key to the genera. Southeast Asian J Trop Med Public Health, 59-79.

35. Rattanarithikul, R., Harbach, R., Harrisson, B., Panthusin, P., Jones, J. \& Coleman, R. (2005) Illustrated keys to the mosquitoes of Thailand; II. Genera culex and lutzia. Southeast Asian J Trop Med Public Health, 37-97.

36. Raymond, H.L. (1987) Action des taons (Diptera: Tabanidae) sur le comportement d'un troupeau de zébus au pâturage en Guyane Française. Ann Zootech 36, 375-386.

37. Raymond, H.L. \& Favre, J. (1991) Essai de lute contre les taons de Gyane Française; effet d'un traitement de détiquage des bovins par aspersion au Butox 50ND sur l'abondance des taons. Rapport INRA/Roussel-UCLAF.

38. Saliba, J., Dandria, D., Grose, J., Harris, E. \& Zammit Lucia, M. (1976) Control of Farm Flies in MaltaIII. The effect of residual insecticide sprays on Musca domestica and Stomoxys calcitrans in the Maltese Islands Pest Managment 22(2), 215-222

39. Sawanon, S., Boonsaen, P. \& Innuruk, P. (2011) Body Measurements of Male Kamphaeng Saen Beef Cattle as Parameters for Estimation of Live Weigh. Kasetsart Journal; Natural Science 45(3), 428434. 
40. Sriwichai, P., Karl, S., Samung, Y., Sumruayphol, S., Kiattibutr, K., Payakkapol, A., Mueller, I., Yan, G., Cui, L. \& Sattabongkot, J. (2015) Evaluation of CDC light traps for mosquito surveillance in a malaria endemic area on the Thai-Myanmar border. Parasit Vectors 8, 636. DOI: 10.1186/s13071-015-1225-3.

41. Taylor, D.B., Moon, R.D. \& Mark, D.R. (2012) Economic impact of stable flies (Diptera: Muscidae) on dairy and beef cattle production. J Med Entomol 49(1), 198-209.

42. Tumrasvin, W. \& Shinonaga, S. (1977) Studies on medically important flies in Thailand. III. Report of species belonging to the genus Musca Linne, including the taxonomic key (Diptera: Muscidae). Bulletin of Tokyo Medical and Dental University 24(3), 209-218.

43. Warnes, M. \& Finlayson, L. (1987) Effect of host behaviour on host preference in Stomoxys calcitrans. Medical and Veterinary entomology 1(1), 53-57.

44. Wickham, H. (2016) ggplot2: Elegant graphics for data analysis. Springer International Publishing., New York,

45. Wiemann, G., Cambell, J., Deshazer, J. \& Berry, I. (1992) Effect of stable flies Diptera muscidae and heat stress on weight gain and feed efficiency of feeder cattle. Journal of economic entomology $\mathbf{8 5}$ 1835-1842.

46. Woo, P.T.K. (1969) The haematocrit centrifuge technique for the detection of Trypanosomes in blood. Can J Zool 47, 921-923.

47. Zaiontz, C.(2019) ANOVA for main and interaction effects. Real statistics using Excel @2012-2019. http://www.real-statistics.com/, WordPress.

48. Zumpt, F. (1973) The Stomoxyine biting flies of the world. Edt Gustave Fischer Verlag Stuttgart, Allemagne, 175p.

\section{Figures}




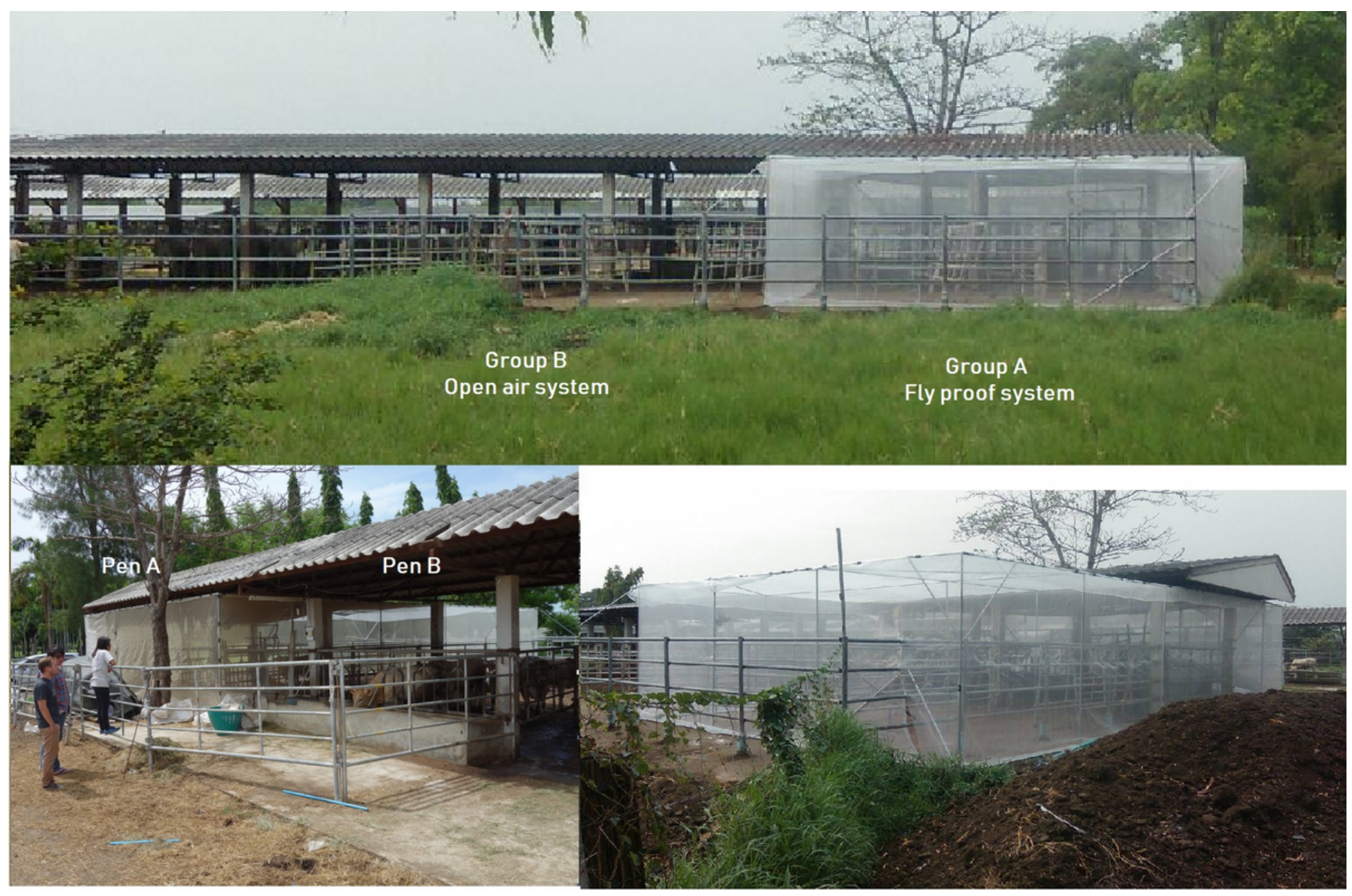

\section{Figure 1}

Pictures of the experimental devices; Group A and Pen A: fly-proof system; Group B and Pen B: open-air system.
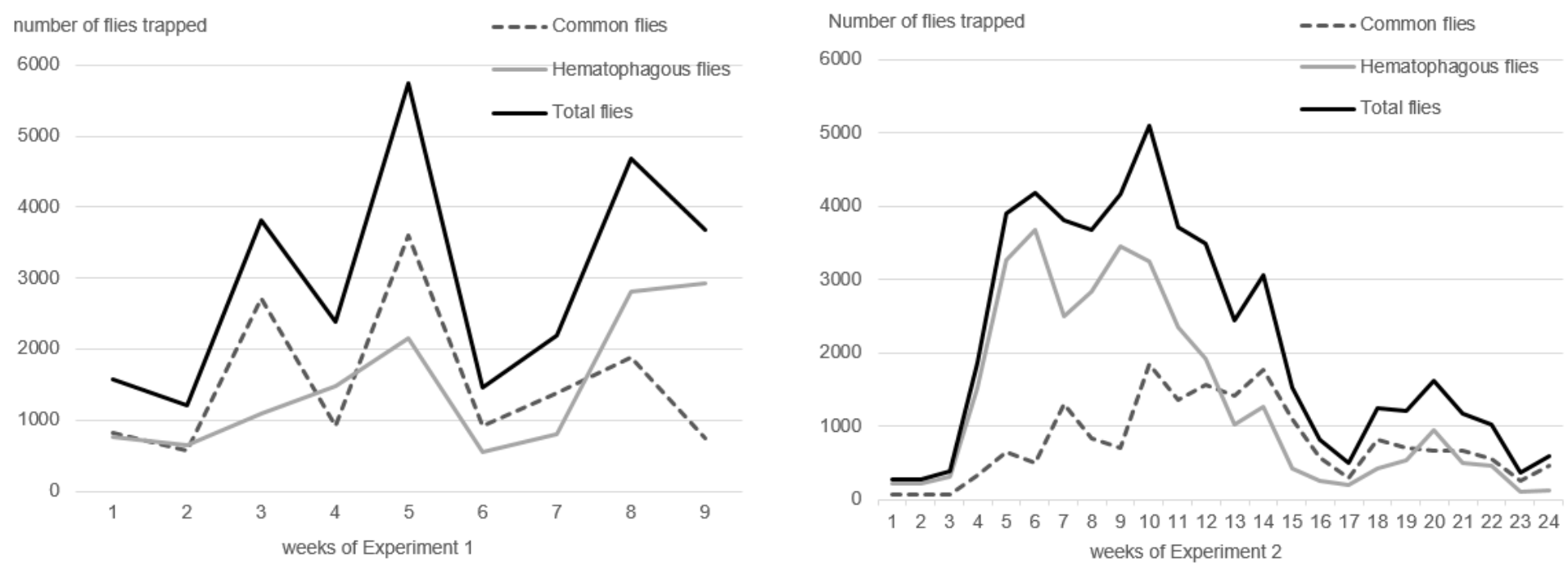

Figure 2

a: Flies densities observed during Experiment 1 b: Flies densities observed during Experiment 2 

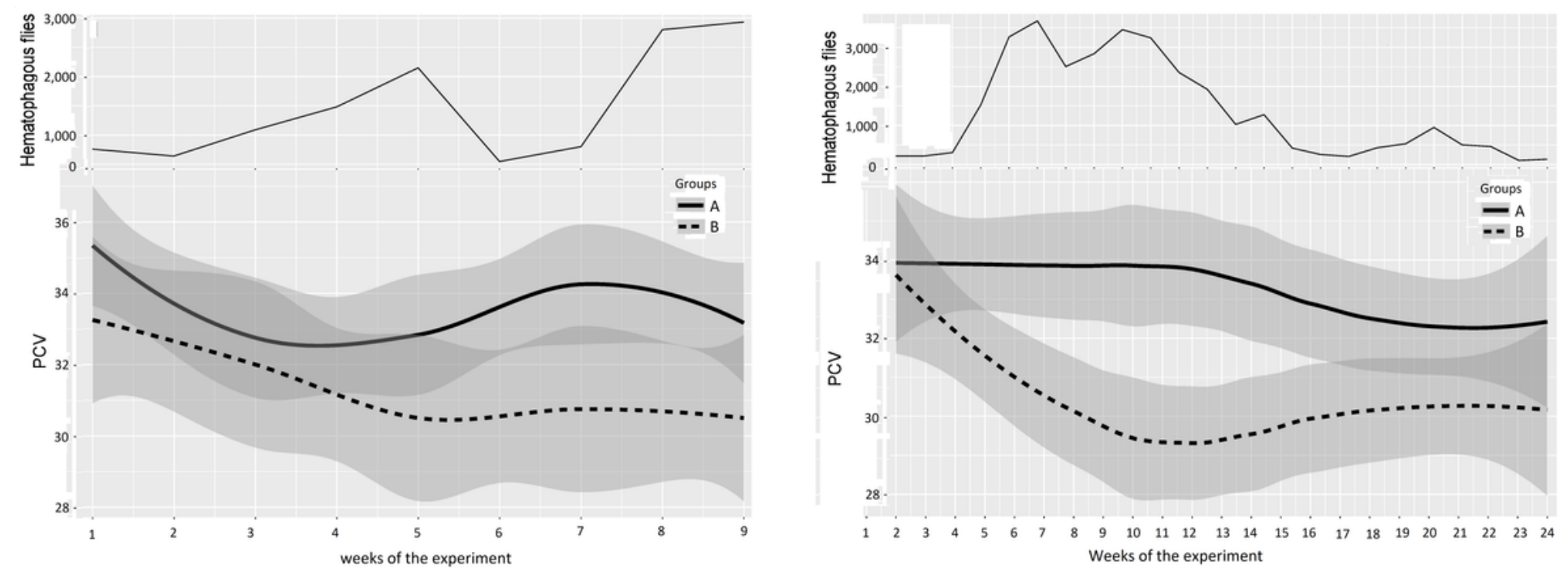

\section{Figure 3}

a: Mean PCV $(95 \% \mathrm{Cl})$ in Groups A and B, and hematophagous flies densities in Experiment 1, from week 1 to 9. Legend: The broad lines indicate smooth mean values, thin solid line for insect density, bold solid line for Group A, bold long-dash for Group B. Shaded parts correspond to the confidence interval of the means (at 95\%). b: Mean PCV $(95 \% \mathrm{Cl})$ in Groups A and B, and hematophagous flies densities in Experiment 2, from week 1 to 24 Legend: The broad lines indicate smooth mean values, thin solid line for insect density, bold solid line for Group A, bold long-dash for Group B. Shaded parts correspond to the confidence interval of the means (at 95\%).
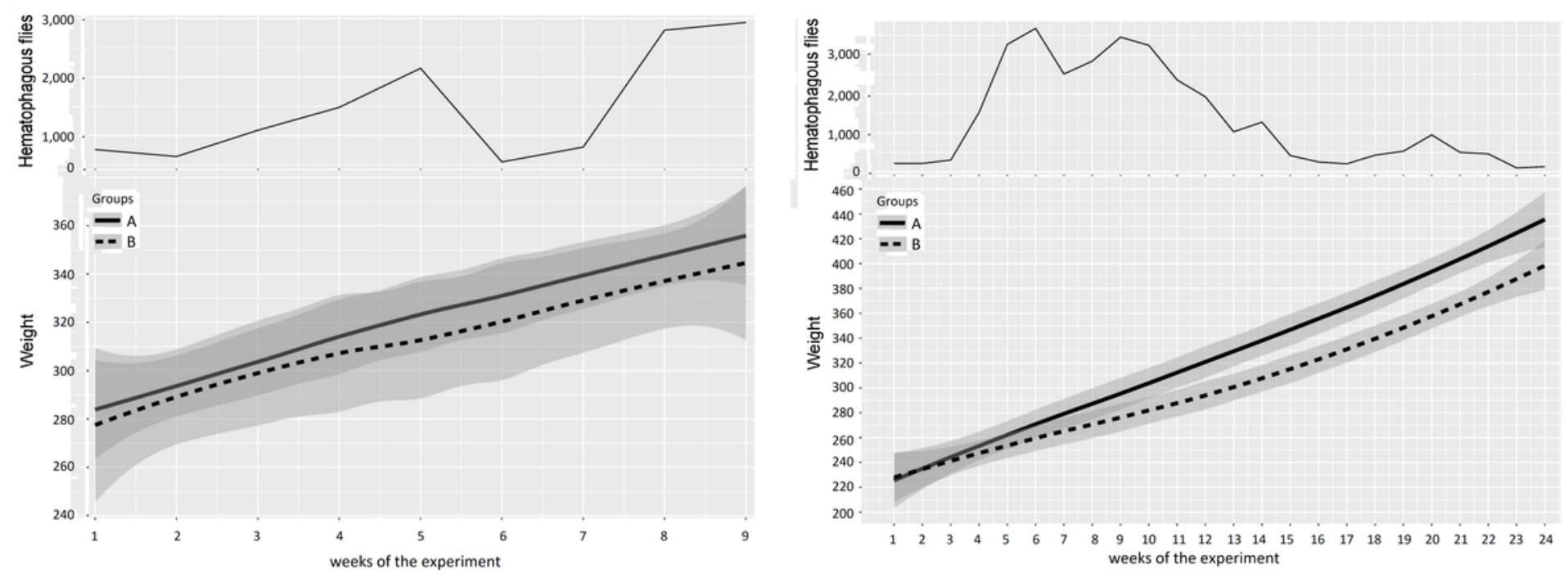

\section{Figure 4}

a: Mean cattle live body weight $(95 \% \mathrm{Cl})$ in Groups $\mathrm{A}$ and $\mathrm{B}$, and hematophagous flies densities in Experiment 1 Legend: The broad lines indicate smooth mean values, thin solid line for insect density, bold solid line for Group A, bold long-dash for Group B. Shaded parts correspond to the confidence interval of the means (at 95\%). b: Mean cattle live body weight $(95 \% \mathrm{Cl})$ in Groups A and B, and hematophagous 
flies densities in Experiment 2 Legend: The broad lines indicate smooth mean values, thin solid line for insect density, bold solid line for Group A, bold long-dash for Group B. Shaded parts correspond to the confidence interval of the means (at 95\%). 